\title{
Consistência na indexação em bibliotecas universitárias brasileiras
}

\author{
Consistency in indexing at Brazilian university libraries
}

Isidoro GIL LEIVA'

Milena Polsinelli RUBI ${ }^{2}$

Mariângela Spotti Lopes FUJITA3

\section{RESUMO}

Objetivou-se avaliar os índices de consistência entre 30 bibliotecas universitárias brasileiras das regiões sul e sudeste por meio de fórmula matemática específica. Como metodologia foi selecionada uma amostra de 30 bibliotecas universitárias que, de acordo com informações em seus sites oficias, dispunham de acervo composto por mais de 100.000 exemplares e permitiam a busca por meio de catálogo on-line. Buscas foram realizadas em cada uma das universidades por meio de seus sites, solicitando livros que continham uma determinada palavra em seu título e publicação em um determinado ano. Como resposta, obtivemos uma lista com os tí́tulos dos documentos disponíveis na biblioteca, na qual escolhemos aleatoriamente um título e solicitamos a visualização do registro completo para comprovação da existência do assunto determinado. Esse procedimento foi repetido até localizarmos cinco bibliotecas com o mesmo título e com os assuntos atribuídos. Como resultado, obtivemos 10 ensaios compostos cada um de 1 quadro e 1 tabela demonstrando as bibliotecas selecionadas, os assuntos encontrados, as linguagens documentárias (ferramentas) e os índices de consistência "relaxado" e "rígido'" Esses ensaios demonstraram grande discrepância entre os valores dos índices de consistência com intervalos entre $73,3 \%$ a $34,4 \%$, no índice relaxado e entre $60 \%$ e $9,6 \%$ no rígido. Verificamos que a coincidência na determinação dos assuntos não é muito elevada, ficando abaixo dos $39 \%$. Concluímos que a diferença entre os índices de consistência pode ser creditada a fatores como: incompatibilidade entre as linguagens documentárias; falta de atualização constante dessas linguagens para acompanhar a evolução do conhecimento; ausência de uma política de indexação bem definida com diretrizes claramente estabelecidas. Procedimentos de indexação seguidos pelos indexadores poderiam contribuir para que o índice de consistência fosse percentualmente maior, uma vez que haveria parâmetros para o processo de indexação.

Palavras-chave: indexação; consistência na indexação; bibliotecas universitárias; Brasil.

\section{ABSTRACT}

The aim of this paper is to evaluate the consistency indexes among 30 Brazilian university libraries from the south and south-east regions through a specific mathematical formula. It was selected a sample of 30 university

\footnotetext{
1 Docente, Departamento de Información y Documentación, Universidad de Murcia, Campus Universitario de Espinardo, Murcia, España. E-mail: <isgil@um.es>.

2 Doutora em Ciência da Informação, Programa de Pós-Graduação em Ciência da Informação, Universidade Estadual Paulista. Marília, SP, Brasil.E-mail: <milena.rubi@gmail.com>.

${ }^{3}$ Docente, Departamento de Ciência da Informação, Universidade Estadual Paulista Júlio de Mesquita Filho. Av. Hygino Muzzi Filho, 737, Campus Universitário, 17525900, Marilia, SP, Brasil. Correspondência para/Correspondece to: M.S.L. FUJITA. E-mail: <goldstar@flash.tv.br>.

Recebido em 3/2/2008 e aceito para publicação em 10/1 1/2008.
} 
libraries that, according to the information in their official sites, have a collection consisted of more than 100.000 copies and allow the search into the on-line catalog. Searches were carried out in every university by means of their sites, requesting books that contained a certain word in its title and were printed in a certain year. The response was a list of available titles in the library, from which we chose at random a title and asked to visualize the complete record to verify the existence of a given subject. This procedure was repeated until we found the same title in five libraries with the chosen subjects. The result is 10 trials, each one consisting of one figure and one table showing the selected libraries, the subjects, the documentary languages (tools) and the consistency indexes "relaxed" and "rigid". These trials show great discrepancy between the values of consistency indexes with intervals between $73,3 \%$ to 34,4\% in the "relaxed" index, and between 60\% and 9,6\% in the "rigid" one. It was revealed that the coincidence in determining the subjects is not too high remaining below $39 \%$. It is concluded that the difference between the consistency indexes may be due to factors as: incompatibility among documentary languages; lack of updating of these languages so as to follow the knowledge evolution; absence of a welldefined indexing policy with guidelines clearly established. Procedures of indexing followed by indexers could contribute to the consistency index to be bigger in percentage, since there would be parameters for the indexing process.

Keywords: indexing; consistency in indexing; Brazilian university libraries.

\section{INTRODUÇÃO}

A consistência na indexação é um elemento característico tanto do processo quanto do resultado do tratamento temático da informação. Ela se caracteriza pelo grau de semelhança na representação da informação documentária de um documento por meio de termos de indexação selecionados por um ou vários indexadores, resultando em um índice de consistência.

Segundo Gil Leiva (2008, p. 72) os elementos que caracterizam o processo, assim como o resultado da indexação, são a exaustividade, a consistência, a especificidade e a correção. Estabelece ainda, que a consistência na indexação foi definida por Zunde e Dexter, dois pioneiros deste assunto, como "[...] o grau de concordância na representação da informação essencial de um documento através de um conjunto de termos de indexação selecionados por cada um dos indexadores de um grupo".

A consistência na indexação pode ser estudada como referência a um único indexador ou a vários. Quando um profissional indexa um mesmo documento, em diferentes momentos temporais, falamos de intraconsistência ou consistência intraindexador. Em segundo lugar, quando vários profissionais indexam um mesmo documento visando comparar seu resultado ou quando se enfrentam duas indexações de diferentes indexadores, falamos de interconsistência ou consistência interindexador.
O resultado da indexação deriva da conjunção de uma série de elementos que se devem tomar em consideração na hora de estudar a consistência. Esses elementos, conforme Figura 1, são:

1) A formação, os conhecimentos na matéria, a profissão e a motivação do indexador;

2) As caraterísticas do objeto indexado, e

3) As condições em que se dá a indexação.

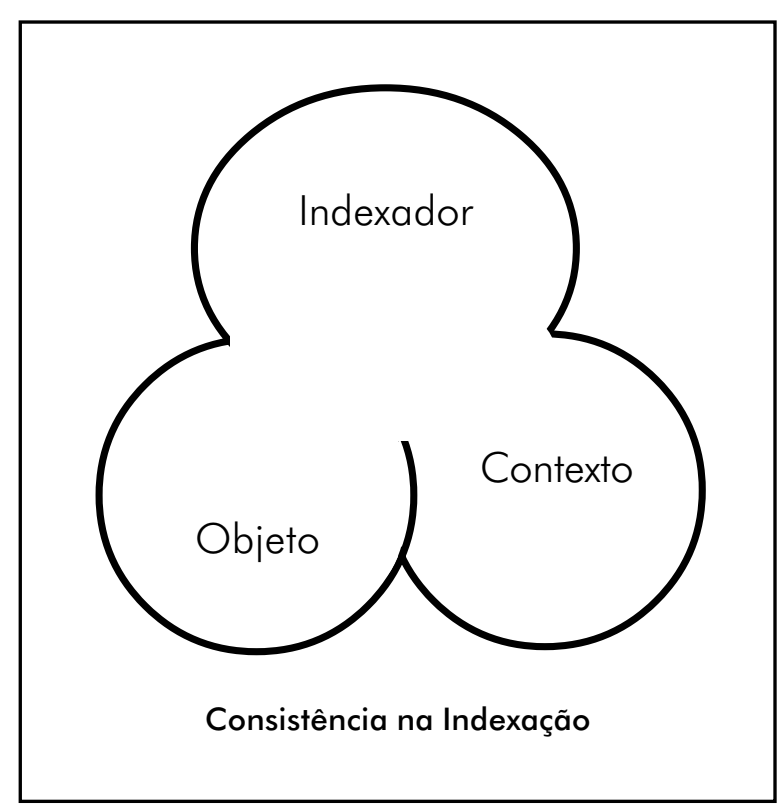

Figura 1. Âmbitos que intervêm na consistência da indexação (GIL LEIVA, 2008, p.74). 
A consistência tem fornecido abundante bibliografia desde a década de 1960 até a atualidade, a qual se pode classificar, por um lado, em literatura teórica, onde se indagam os motivos que provocam a consecução de resultados diferentes na indexação, e, por outro lado, em literatura mais experimental, que procura quantificar a semelhança entre várias indexações. No primeiro grupo, os pesquisadores têm trabalhado nos fatores que intervêm durante o processo, as habilidades e as metodologias leitoras dos indexadores, a relação entre a seleção de conceitos e a recuperação, as causas que levam os indexadores a escolher ou rejeitar termos em função de suas propriedades, assim como os aspectos psicológicos que intermediam durante a indexação.
A outra parte importante da literatura está dedicada à quantificação da consistência, ou seja, à obtenção de índices de consistência que vão desde o 1 ao $100 \%$, mediante fórmulas diversas de semelhança entre indexações, apoiando-se em diferentes aspectos como a experiência (indexadores principiantes ou indexadores experientes) ou as tipologias documentais (livros, patentes, artigos de revista, fotografias, etc). Fazer comparações entre indexadores é um assunto complicado. Quando se pretende comparar a indexação de uma instituição com outra, é preciso controlar ao máximo o número de elementos que, em maior ou menor medida, afeta seu resultado (Quadro 1).

Quadro 1. Elementos que devem ser considerados na comparação de indexações.

\begin{tabular}{|c|c|}
\hline $\begin{array}{l}\text { I } \\
\text { D } \\
\text { D } \\
\text { E } \\
X \\
\text { A } \\
\text { D } \\
\text { O } \\
\text { R }\end{array}$ & $\begin{array}{l}\text { - Formação e experiência em indexação: indexador experiente contra principiante } \\
\text { - Conhecimento da matéria } \\
\text { - Domínio das ferramentas da indexação (linguagens de indexação) } \\
\text { - Profissionalismo }\end{array}$ \\
\hline $\begin{array}{l}\text { C } \\
\text { O } \\
\text { N } \\
\text { T } \\
\text { E } \\
X \\
\text { T } \\
\text { O }\end{array}$ & $\begin{array}{l}\text { - Políticas de indexação marcadas pela instituição } \\
\text { - Objetivo da indexação: temas principais contra especificidade } \\
\text { - Tipos e necessidades dos usuários } \\
\text { - Carga de trabalho e tempo dedicado }\end{array}$ \\
\hline $\begin{array}{l}\text { O } \\
\text { B } \\
\text { J } \\
\text { E } \\
\text { T } \\
\text { O }\end{array}$ & $\begin{array}{l}\text { - Complexidade do objeto indexado: livro infantil contra patentes } \\
\text { - Características e propriedades do objeto indexado: material textual contra material gráfico } \\
\text { ou audiovisual } \\
\text { - Tamanho: indexação de textos curtos contra textos compridos }\end{array}$ \\
\hline $\begin{array}{c}M \\
O \\
M \\
E \\
N \\
T \\
O\end{array}$ & $\begin{array}{l}\text { - A comparação executa-se com as palavras } \\
\text { - chave pinçadas diretamente do texto ou também, uma vez convertidas essas palavras- } \\
\text { chave em descriptores, após sua filtragem com o vocabulário controlado }\end{array}$ \\
\hline $\begin{array}{l}F \\
\text { Ó } \\
R \\
M \\
U \\
L \\
A\end{array}$ & - São variadas as fórmulas matemáticas utilizadas para conseguir os índices de consistência \\
\hline
\end{tabular}

Fonte: Gil Leiva, 2008, p. 75. 
Segundo Gil Leiva (2008, p. 385) pode-se levar a cabo uma avaliação intrínseca e extrínseca da indexação. A avaliação intrínseca da indexação é

[...] o conjunto de tarefas centradas no resultado da indexação (descritores, cabeçalhos, sub-cabeçalhos ou identificadores) com a finalidade de conhecer sua qualidade. A avaliação intrínseca da indexação pode ser qualitativa, isto é, por meio de valorações e consensos entre os experientes, ou quantitativa, mediante fórmulas.
A avaliação intrínseca quantitativa procura conhecer o grau de semelhança entre os indexadores. Por esse motivo, o grau de consistência será maior quanto mais semelhantes sejam as indexações. Comparadas, as categorias oscilam de 0 a 1 ou de 0 a 100 , quando se traslada a um \%. Essa semelhança ou diferença entre indexações pode-se quantificar por meio de fórmulas matemáticas. Duas equações para calcular os índices de consistência entre duas indexações são as seguintes (Quadro 2):

Quadro 2. Equações de índices de consistência.

\begin{tabular}{|c|c|}
\hline Hooper (1965) & Rolling (1981) \\
\hline$\frac{C}{A+B-C}$ & $\frac{2 C}{A+B}$ \\
Uma variante dessa equação é: & onde, \\
$\qquad \frac{100 C}{C+A+B}$ & $\mathrm{C}=$ Termos comuns nas duas indexações \\
& $\mathrm{A}=$ Termos usados na indexação $\mathrm{A}$ \\
onde, & $\mathrm{B}=$ Termos usados na indexação $\mathrm{B}$ \\
$\mathrm{C}=$ Termos comuns nas duas indexações & \\
$\mathrm{A}=$ Termos usados na indexação A mas não na $\mathrm{B}$ & \\
$\mathrm{B}=$ Termos usados na indexação $\mathrm{B}$ mas não na $\mathrm{A}$ & \\
\end{tabular}

Uma variante da fórmula de Hooper, que tem sido utilizada em diferentes lugares por Gil Leiva (1997, 1999, 2001, 2002 e 2008,) é a seguinte:

$$
C_{i}=\frac{T_{c o}}{(A+B)-T_{c o}}
$$

onde,

$T_{c o}=$ Número de termos comuns nas duas indexações

$A^{c o}=$ Número de termos usados na indexação $A$

$B=$ Número de termos usados na indexação $B$

Para finalizar essa parte introdutória a respeito da consistência na indexação, é importante mencionar que "[...] da revisão dos resultados obtidos nos experimentos levados a cabo nos últimos anos, depreende-se que a média dos índices oscila entre os $25 \%$ e os $60 \%$ de coincidências. Uma análise minuciosa do que foi dito nos parágrafos anteriores, juntamente aos dados mencionados, fazem-nos assumir que a inconsistência é uma caraterística inerente à indexação, e não usa anomalia esporádica." (Gil Leiva, 2008, p.76).

O objetivo deste artigo é encontrar índices de consistência entre 30 bibliotecas universitárias localizadas nas regiões do sudeste e sul do Brasil, por meio da fórmula matemática apresentada anteriormente. Em continuidade, explica-se a metodologia adotada.

\section{METODOLOGIA}

De acordo com dados do ano de 2006, fornecidos pelo Ministério da Educação (MEC), pelo Instituto Nacional de Estudos e Pesquisas Educacionais 
Anísio Teixeira (INEP) e pela Diretoria de Estatísticas e Avaliação da Educação Superior (DEAES), estão instaladas no Brasil 2270 universidades distribuídas pelas regiões do território nacional da seguinte maneira:

- norte: 135;

- nordeste: 412;

- sudeste: 1093;

- centro-oeste: 243;

- sul: 387.

Tendo em vista o grande número de universidades instaladas no Brasil, utilizamos como critério para seleção, primeiramente, aquelas situadas nas regiões sudeste e nordeste, pelo fato de possuírem o maior número de universidades. Porém, ao procurarmos seus catálogos on-line, observamos que nem todas as bibliotecas universitárias da região nordeste disponibilizavam esse serviço, o que impossibilitaria esta pesquisa. Essa situação pode ser explicada, em parte, pela recente expansão das universidades naquela região. Entendemos que as universidades e suas bibliotecas estão passando por um período de consolidação. Nossa afirmativa encontra respaldo em documento do Ministério da Educação (Brasil, 2005) que esclarece que o maior crescimento percentual de instituições registrado no ano de 2005 se encontra nas regiões norte $(16,8 \%)$ e nordeste $(13,2 \%)$ e o mais baixo na região centro-oeste, com um crescimento de apenas $2,4 \%$.

Dessa forma, nossa amostra é composta por 30 bibliotecas universitárias situadas nas regiões sudeste e sul que, de acordo com informações em seus sites oficiais, dispunham de acervo composto por mais de 100.000 exemplares e permitiam a busca por meio de catálogo on-line.

Para averiguação da consistência na indexação em bibliotecas universitárias brasileiras, foram realizadas buscas por assunto nos catálogos on-line disponibilizados pelas seguintes universidades (Quadro 3):

Quadro 3. Universidades utilizadas na amostra.

\begin{tabular}{|c|c|}
\hline ESTADO & UNIVERSIDADE \\
\hline Espírito Santo & Universidade Federal do Espírito Santo \\
\hline Minas Gerais & $\begin{array}{l}\text { Universidade Federal de Viçosa (UFV) } \\
\text { Pontifícia Universidade Católica de Minas Gerais (PUC-MG) } \\
\text { Universidade Federal de Minas Gerais (UFMG) } \\
\text { Universidade Federal de Uberlândia }\end{array}$ \\
\hline Paraná & $\begin{array}{l}\text { Universidade Federal do Paraná (UFPR) } \\
\text { Universidade Estadual do Centro-Oeste (UNICENTRO) } \\
\text { Universidade Estadual de Londrina (UEL) } \\
\text { Pontifícia Universidade Católica (PUC-PR) } \\
\text { Universidade Estadual de Maringá }\end{array}$ \\
\hline Rio de Janeiro & $\begin{array}{l}\text { Pontifícia Universidade Católica do Rio de Janeiro } \\
\text { Universidade Federal do Rio de Janeiro } \\
\text { Centro Universitário da Cidade }\end{array}$ \\
\hline Rio Grande do Sul & $\begin{array}{l}\text { Universidade Federal do Rio Grande do Sul (UFRGS) } \\
\text { Universidade de Caxias do Sul (UCS)Universidade da Região do Vale do Rio dos Sinos } \\
\text { (UNISINOS) } \\
\text { Universidade Federal de Pelotas } \\
\text { Universidade Federal de Santa Maria } \\
\text { Centro Universitário Franciscano } \\
\text { Universidade Regional do Noroeste do Estado do Rio Grande do Sul (UNIJUÍ) }\end{array}$ \\
\hline Santa Catarina & $\begin{array}{l}\text { Universidade Regional de Blumenau (FURB/SC) } \\
\text { Universidade Federal de Santa Catarina (UFSC) } \\
\text { Universidade do Estado de Santa Catarina (UDES) } \\
\text { UNIPLAC - Fundação das Escolas Unidas do Planalto Catarinense }\end{array}$ \\
\hline São Paulo & $\begin{array}{l}\text { Faculdade de Direito Milton CamposUniversidade São Francisco (USF) } \\
\text { Universidade Estadual de Campinas (UNICAMP) } \\
\text { Pontifícia Universidade Católica de Campinas (PUC-Campinas) } \\
\text { Universidade Estadual Paulista Júlio de Mesquita Filho (UNESP) }\end{array}$ \\
\hline
\end{tabular}


Como não há um único catálogo brasileiro online que reúna todos os catálogos das universidades brasileiras, uma série de buscas foi realizada em cada uma das universidades por meio de seus sites. Nessas pesquisas, solicitamos livros que continham uma determinada palavra em seu título e publicação em um determinado ano, por exemplo, "anatomia" no campo Título e "2000" no campo Ano de publicação. Decidimos que no campo Ano de publicação ficariam representados todos os anos desde 1997 a 2006.

As palavras dos títulos foram escolhidas aleatoriamente, mas de modo a contemplar as três áreas do conhecimento: Exatas, Humanas e Biológicas.

Em resposta a essas pesquisas, obtivemos uma lista com os títulos dos documentos disponíveis na biblioteca. Em seguida, escolhemos aleatoriamente um título e solicitamos a visualização do registro completo para comprovação da existência do assunto determinado. Esse procedimento foi repetido até localizarmos cinco bibliotecas com o mesmo título e com os assuntos atribuídos. Essas duas últimas etapas foram repetidas várias vezes até conseguir os dez ensaios demonstrados em APÊNDICE.

Posteriormente, adotou-se a metodologia utilizada por Gil Leiva (2001) para a comparação de indexações entre Bibliotecas Públicas espanholas.

Para isto, empregou-se, portanto, a fórmula já mencionada:

$$
C_{i}=\frac{T_{c o}}{(A+B)-T_{c o}}
$$

Além disso, foram praticados dois tipos de comparações para chegarmos aos índices de consistência: utilizadas para a "tradução" da síntese dos textos, ou seja, as ferramentas utilizadas para essa finalidade. Por se tratar de um estudo realizado utilizando metodologia semelhante à de Gil Leiva (1998), utilizaremos também neste artigo o termo "ferramenta" na terceira coluna de todos os quadros (APÊNDICE), para designarmos as linguagens documentárias.

\section{RESULTADOS E DISCUSSÃO}

Os resultados obtidos foram organizados no que denominamos de ensaio. Cada um dos ensaios é constituído por um quadro e uma tabela. No total, foram realizados 10 ensaios compostos por 10 quadros e 10 tabelas, totalizando 20 (APÊNDICE).

Cada quadro dos ensaios é composto por 3 colunas e 6 linhas. Na primeira coluna estão relacionados os nomes de cinco bibliotecas que foram utilizadas para análise de consistência naquele ensaio, ou seja, as bibliotecas em que o mesmo livro foi localizado. Na coluna a seguir estão representados os assuntos dos livros designados pelas bibliotecas que foram copiados do campo "assunto" dos registros bibliográficos dos seus catálogos on-line. Na terceira e última coluna estão relacionadas às ferramentas utilizadas pelos bibliotecários para a "tradução" da síntese dos textos, ou seja, as linguagens documentárias utilizadas. Essa observação foi possível naqueles catálogos que disponibilizavam o registro completo no formato MARC (Machine Readable Cataloging). Para aquelas bibliotecas que não disponibilizavam essa informação, quinze no total, foi enviado um e-mail solicitando esclarecimento. Obtivemos resposta de seis bibliotecas. Nos quadros dos ensaios (APÊNDICE), quando não há informação sobre a ferramenta utilizada, indicamos da seguinte forma: "NÃO INFORMADO". Esclarecemos, no entanto, que não podemos afirmar se não existe ou se não há utilização de uma linguagem documentária para a tradução das sínteses dos assuntos. Afirmamos apenas que essa informação não foi informada, seja pelo site da instituição ou pelo email enviado.

Para fins de complementação, acima de cada quadro há a indicação da palavra solicitada no campo de busca "título", o ano de publicação do livro e a referência do livro encontrado na busca. 
Após cada quadro há, também, uma tabela que compõe cada um dos ensaios. Essa tabela é formada por 3 colunas e 12 linhas. Na primeira coluna estão relacionadas às bibliotecas selecionadas para o ensaio apresentado no quadro anterior, organizadas em pares, de modo a possibilitar o cálculo do índice de consistência entre elas. Ressaltamos que os pares de bibliotecas foram escolhidos aleatoriamente, sendo selecionadas aquelas em que foram encontrados os

Tabela 1. Índice de consistência acumulado dos 10 ensaios - relaxado.

\begin{tabular}{l|c}
\hline ENSAIOS & $\begin{array}{c}\text { ÍNDICE DE CONSISTÊNCIA } \\
\text { (RELAXADO) }\end{array}$ \\
\hline Ensaio 4 & 73,3 \\
\hline Ensaio 1 & 55,8 \\
\hline Ensaio 5 & 39,2 \\
\hline Ensaio 9 & 36,9 \\
\hline Ensaio 7 & 36 \\
\hline Ensaio 10 & 25,1 \\
\hline Ensaio 8 & 23,7 \\
\hline Ensaio 6 & 21,8 \\
\hline Ensaio 3 & 18,5 \\
\hline Ensaio 2 & 13,7 \\
\hline Média & $\mathbf{3 4 , 4}$ \\
\hline
\end{tabular}

Organizando os resultados em ordem decrescente, observamos uma grande discrepância entre a primeira e a última linha da tabela. No caso da Tabela 1, em que estão demonstrados os índices de consistência relaxados, há um intervalo que varia entre $73,3 \%$ a $34,4 \%$. Na Tabela 2, em que foram verificados os índices de consistência rígidos, esse intervalo é ainda maior, variando entre $60 \%$ e $9,6 \%$.

Observando os resultados das tabelas (APÊNDICE), verificamos que a coincidência na determinação dos assuntos não é muito elevada, ficando abaixo dos 39\%, com exceção apenas do ensaio 4 que obteve, no índice relaxado, $73 \%$ de coincidência.

Deve-se levar em consideração que, na maioria dos casos, as linguagens documentárias utilizadas são diferentes. Além disso, a maioria não faz parte de uma livros com o mesmo título e com assuntos atribuídos a eles. Nas duas colunas seguintes estão apresentados os índices de consistência "relaxados" e "rígidos" obtidos pela avaliação extrínseca entre pares de bibliotecas participantes em cada ensaio (APÊNDICE).

A seguir, elaboramos duas tabelas com os índices acumulados dos 10 ensaios, na qual verificamos que o índice de consistência relaxado é de 34,4 e o índice de consistência rígido é de 27,3.

Tabela 2. Índice de consistência acumulado dos 10 ensaios - rígido.

\begin{tabular}{c|c}
\hline ENSAIOS & $\begin{array}{c}\text { ÍNDICE DE CONSISTÊNCIA } \\
\text { (RÍGIDO) }\end{array}$ \\
\hline Ensaio 4 & 60 \\
\hline Ensaio 1 & 55,8 \\
\hline Ensaio7 & 32,4 \\
\hline Ensaio 5 & 29 \\
\hline Ensaio 9 & 28,3 \\
\hline Ensaio 10 & 20 \\
\hline Ensaio 3 & 17,9 \\
\hline Ensaio 6 & 10 \\
\hline Ensaio 8 & 10 \\
\hline Ensaio 2 & 9,6 \\
\hline Média & $\mathbf{2 7 , 3}$ \\
\hline
\end{tabular}

rede que compartilha os registros bibliográficos e se comprometem a utilizar uma linguagem documentária específica.

A consistência da indexação está ligada a dois elementos: ao desempenho do indexador e à qualidade dos instrumentos de indexação.

Quanto ao desempenho do indexador, é necessário destacar que o tempo é uma variável com a qual o profissional deve lidar para desempenhar sua tarefa. Além disso, é importante que haja adoção de diretrizes de política de indexação, como, por exemplo,número de termos para o documento, que o auxiliarão na imparcialidade no momento da determinação do assunto. Com isso, procura-se controlar a subjetividade, inerente a qualquer trabalho humano e presente na atividade de indexação. 
Ressaltamos que um índice de consistência alto é difícil de ser obtido em casos como o do nosso estudo, em que as pessoas trabalham em lugares diferentes.

Na literatura publicada em Ciência da Informação no Brasil, destacamos o estudo de consistência de González et al. (1998) sobre avaliação de repertórios brasileiros em Agricultura, Ciência da Informação e Direito, que utilizou método para avaliação de índice de consistência.

Gil Leiva (1998) realizou estudo semelhante, verificando o índice de consistência em bibliotecas públicas na Espanha. Seu estudo demonstrou que esse índice variava entre $46,6 \%$ no relaxado e $37,7 \%$ no rígido.

Comparando com nosso estudo, verificamos que existem diferenças significativas entre as indexações das bibliotecas. Acreditamos que isso se deve à falta de compatibilidade entre as linguagens documentárias utilizadas, uma vez que cada biblioteca utiliza uma linguagem distinta. Além disso, há também a utilização de linguagens que não refletem a realidade brasileira, pois são traduções de versões em inglês, por exemplo. Observamos também que uma política de indexação insuficiente ou inexistente pode contribuir para a falta de sistematização dos procedimentos de indexação e das diretrizes a serem seguidas pelos bibliotecários durante a realização da indexação. Finalmente, a ausência de avaliação e divulgação de resultados da recuperação da informação refletirão na consistência entre indexadores de diferentes bibliotecas.

\section{CONSIDERAÇÕES FINAIS}

Os resultados obtidos demonstram que a consistência na indexação de catálogos de bibliotecas universitárias do Brasil encontra-se entre 34 e 27 \%. Comparados com os resultados obtidos por Gil Leiva (2001) em catálogos de bibliotecas públicas da Espanha, que foram de 46,6\% e 37,7\%, podemos observar que estes índices de consistência foram maiores.

Acreditamos que esse fato se justifica, principalmente, devido ao fato de haver um único site (www.mcu.es/bpe/bpe.html) por meio do qual é possível acessar os catálogos de cada uma das bibliotecas espanholas, o que facilita as buscas.
Além disso, há uma coincidência maior entre as ferramentas utilizadas para a tradução dos assuntos dos documentos. A maioria das bibliotecas públicas espanholas utiliza a "Lista de Encabezamientos de Materia para Bibliotecas Públicas" (LEM-BP) e Rebeca (Bases de dados) fazendo com que haja um maior índice de consistência entre elas.

Isso demonstra uma preocupação com a qualidade da informação recuperada e o compromisso em trabalhar sob a forma de cooperação, dando indícios de uma política de indexação definida.

Essa diferença entre os índices de consistência de bibliotecas universitárias pode ser creditada à incompatibilidade entre as linguagens documentárias, no que diz respeito tanto à linguagem do usuário quanto à linguagem do autor. As linguagens documentárias, que muitas vezes se constituem em uma tradução de linguagens estrangeiras, não refletem as características culturais e linguísticas brasileiras. Além disso, sabemos das dificuldades de uma atualização constante das linguagens documentárias para acompanhar a evolução do conhecimento.

Consideramos que uma política de indexação bem definida - com diretrizes claramente estabelecidas e procedimentos de indexação seguidos pelos indexadores -, poderia contribuir para que o índice de consistência fosse percentualmente maior, uma vez que haveria parâmetros para o processo de indexação.

Todavia, há de se considerar a afirmação de Gil Leiva (2008) quanto à característica de inconsistência ser inerente à indexação. Além disso, a média dos índices de consistência na indexação de bibliotecas universitárias oscilou entre 34 e $27 \%$, o que os inclui na média dos índices que oscilam entre os $25 \%$ e os $60 \%$ de coincidências obtidos em resultados de estudos revistos por Gil Leiva (2008).

Ainda assim, há carência de trabalhos no Brasil que possam ser utilizados em comparação com esta pesquisa. Acreditamos que outros estudos sobre avaliação da recuperação da informação devam ser realizados pelas bibliotecas universitárias com vistas não só à comparação de resultados, mas principalmente à melhoria dos índices de consistência que serão reflexos de uma indexação de qualidade. 
BRASIL. Ministério da Educação. Censo da educação superior 2004: resumo técnico. Brasília, DF, 2005. Disponível em: <http:/ /www.inep.gov.br/download/superior/2004/censosuperior/ Resumo tecnico-Censo 2004.pdf>. Acesso em: 26 ago. 2006.

GIL LEIVA, I. Manual de indización: teoría y práctica. Gijón: Trea, 2008.

GIL LEIVA, I. Consistencia en la asignación de materias en bibliotecas públicas del Estado. Boletín de la Asociación Andaluza de Bibliotecarios, n.63, p.69-96, 2001. Disponível em: <http:// webs.um.es/isgil>. Acesso em: 17 set. 2008.
INSTITUTO NACIONAL DE ESTUDOS E PESQUISAS EDUCACIONAIS ANÍSIO TEIXEIRA. Sinopses estatísticas da educação superior: graduação. Brasília, DF, 2006. Disponível em: <http:/ /www.inep.gov.br/superior/censosuperior/sinopse/>. Acesso em: 26 ago. 2006.

GONZÁLEZ, J.A.M. et al. Avaliação de repertórios brasileiros em agricultura, ciência da informação e direito: uma análise de conteúdo. Ciência da Informação, v.27, n.3, set./ dez. 1998. Disponível em: <http://revista.ibict.br/index.php/ ciinf/article/viewFile/31 1/277 > . Acesso em: 29 ago. 2008. 

ENSAIOS PARA ESTUDOS DE ÍNDICES DE CONSISTÊNCIA

\section{ENSAIO 1}

Busca: Título 'construtivismo'; ano de publicação '1997'.

Livro encontrado na busca: COLL, C. O construtivismo em sala de aula. São Paulo: Ática, 1997.

Quadro 1. Ensaio 1 - "Construtivismo".

\begin{tabular}{|c|c|c|}
\hline BIBLIOTECAS & ASSUNTO & FERRAMENTAS UTILIZADAS \\
\hline $\begin{array}{c}\text { Pontifícia Universidade } \\
\text { Católica do Rio de Janeiro }\end{array}$ & Construtivismo (educação) & BIBLIODATA \\
\hline $\begin{array}{c}\text { Universidade Federal do Rio de } \\
\text { Janeiro }\end{array}$ & $\begin{array}{c}\text { Psicologia educacional } \\
\text { Construtivismo (educação) } \\
\text { Psicologia da aprendizagem }\end{array}$ & BIBLIODATA \\
\hline $\begin{array}{c}\text { Universidade Federal do } \\
\text { Espírito Santo }\end{array}$ & $\begin{array}{c}\text { Construtivismo (educação) } \\
\text { Aprendizagem }\end{array}$ & BIBLIODATA \\
\hline $\begin{array}{c}\text { Universidade Federal do } \\
\text { Paraná (UFPR) }\end{array}$ & $\begin{array}{c}\text { Construtivismo (educação) } \\
\text { Aprendizagem }\end{array}$ & BIBLIODATA \\
\hline $\begin{array}{c}\text { Universidade Estadual de } \\
\text { Londrina (UEL) }\end{array}$ & $\begin{array}{c}\text { Construtivismo } \\
\text { (educação)Aprendizagem }\end{array}$ & \\
\hline
\end{tabular}

Tabela 1. Índice de consistência entre pares de bibliotecas do Ensaio 1.

\begin{tabular}{|c|c|c|}
\hline PARES DE BIBLIOTECAS & $\begin{array}{c}\text { ÍNDICE DE } \\
\text { CONSISTÊNCIA } \\
\text { (RELAXADO) }\end{array}$ & $\begin{array}{c}\text { ÍNDICE DE } \\
\text { CONSISTÊNCIA } \\
\text { (RÍGIDO) }\end{array}$ \\
\hline $\begin{array}{l}\text { Pontifícia Universidade Católica do Rio de Janeiro - Universidade } \\
\text { Federal do Rio de Janeiro }\end{array}$ & 33,33 & 33,3 \\
\hline $\begin{array}{l}\text { Pontifícia Universidade Católica do Rio de Janeiro - Universidade } \\
\text { Federal do Espírito Santo }\end{array}$ & 50 & 50 \\
\hline $\begin{array}{l}\text { Pontifícia Universidade Católica do Rio de Janeiro - Universidade } \\
\text { Federal do Paraná (UFPR) }\end{array}$ & 50 & 50 \\
\hline $\begin{array}{c}\text { Pontifícia Universidade Católica do Rio de Janeiro - Universidade } \\
\text { Estadual de Londrina (UEL) }\end{array}$ & 50 & 50 \\
\hline $\begin{array}{l}\text { Universidade Federal do Rio de Janeiro - Universidade Federal do } \\
\qquad \text { Espírito Santo }\end{array}$ & 25 & 25 \\
\hline $\begin{array}{l}\text { Universidade Federal do Rio de Janeiro - Universidade Federal do } \\
\text { Paraná (UFPR) }\end{array}$ & 25 & 25 \\
\hline $\begin{array}{l}\text { Universidade Federal do Rio de Janeiro - Universidade Estadual de } \\
\text { Londrina (UEL) }\end{array}$ & 25 & 25 \\
\hline $\begin{array}{l}\text { Universidade Federal do Espírito Santo - Universidade Federal do } \\
\text { Paraná (UFPR) }\end{array}$ & 100 & 100 \\
\hline $\begin{array}{l}\text { Universidade Federal do Espírito Santo - Universidade Estadual de } \\
\text { Londrina (UEL) }\end{array}$ & 100 & 100 \\
\hline $\begin{array}{l}\text { Universidade Federal do Paraná (UFPR) - Universidade Estadual de } \\
\text { Londrina (UEL) }\end{array}$ & 100 & 100 \\
\hline MÉDIA & 55,8 & 55,8 \\
\hline
\end{tabular}




\section{ENSAIO 2}

Busca: Título 'crime'; ano de publicação '1998'.

Livro encontrado na busca: HERKENHOFF, J. B. Crime: tratamento sem prisão - relato da experiencia de uma justiça criminal alternativa. Porto Alegre: Livraria do Advogado, 1998.

Quadro 2. Ensaio 2 - "Crime".

\begin{tabular}{|c|c|c|}
\hline BIBLIOTECAS & ASSUNTO & FERRAMENTAS UTILIZADAS \\
\hline $\begin{array}{l}\text { Pontifícia Universidade Católica } \\
\text { (PUC - PR) }\end{array}$ & $\begin{array}{l}\text { Crime e criminosos } \\
\text { Reincidentes (Delito) } \\
\text { Pena (Direito) } \\
\text { Direito Penal } \\
\text { Prevenção do crime }\end{array}$ & Library of Congress Subject Headings \\
\hline $\begin{array}{l}\text { Universidade Federal de Minas } \\
\text { Gerais (UFMG) }\end{array}$ & $\begin{array}{l}\text { Crime e criminosos } \\
\text { Reincidentes }\end{array}$ & NÃO INFORMADO \\
\hline Universidade São Francisco (USF) & $\begin{array}{c}\text { Pena sem prisão } \\
\text { Brasil } \\
\text { Direito }\end{array}$ & NÃO INFORMADO \\
\hline $\begin{array}{c}\text { Pontifícia Universidade Católica } \\
\text { do Rio de Janeiro }\end{array}$ & $\begin{array}{c}\text { Pena (Direito) } \\
\text { Reabilitação de criminosos } \\
\text { Crime e criminosos }\end{array}$ & BIBLIODATA \\
\hline $\begin{array}{l}\text { Universidade Federal do Rio } \\
\text { Grande do Sul (UFRGS) }\end{array}$ & $\begin{array}{c}\text { Direito Penal } \\
\text { Prisão } \\
\text { Presidiários (Direito) } \\
\text { Direito penitenciário }\end{array}$ & Não utiliza nenhuma linguagem \\
\hline
\end{tabular}

Tabela 2. Índice de consistência entre pares de bibliotecas do Ensaio 2.

\begin{tabular}{|c|c|c|}
\hline PARES DE BIBLIOTECAS & $\begin{array}{c}\text { ÍNDICE DE } \\
\text { CONSISTÊNCIA } \\
\text { (RELAXADO) }\end{array}$ & $\begin{array}{c}\text { ÍNDICE DE } \\
\text { CONSISTÊNCIA } \\
\text { (RÍGIDO) }\end{array}$ \\
\hline $\begin{array}{l}\text { Pontifícia Universidade Católica (PUC - PR) - } \\
\text { Universidade Federal de Minas Gerais (UFMG) }\end{array}$ & 27,2 & 16,6 \\
\hline $\begin{array}{c}\text { Pontifícia Universidade Católica (PUC - PR) - } \\
\text { Universidade São Francisco (USF) }\end{array}$ & 6,6 & 0 \\
\hline $\begin{array}{c}\text { Pontifícia Universidade Católica (PUC - PR) - Pontifícia } \\
\text { Universidade Católica do Rio de Janeiro }\end{array}$ & 33,3 & 33,3 \\
\hline $\begin{array}{c}\text { Pontifícia Universidade Católica (PUC - PR) - } \\
\text { Universidade Federal do Rio Grande do Sul (UFRGS) }\end{array}$ & 12,5 & 12,5 \\
\hline $\begin{array}{c}\text { Universidade Federal de Minas Gerais (UFMG) - } \\
\text { Universidade São Francisco (USF) }\end{array}$ & 0 & 0 \\
\hline $\begin{array}{l}\text { Universidade Federal de Minas Gerais (UFMG) - } \\
\text { Pontifícia Universidade Católica do Rio de Janeiro }\end{array}$ & 25 & 25 \\
\hline $\begin{array}{l}\text { Universidade Federal de Minas Gerais (UFMG) - } \\
\text { Universidade Federal do Rio Grande do Sul (UFRGS) }\end{array}$ & 0 & 0 \\
\hline $\begin{array}{l}\text { Universidade São Francisco (USF) - Pontifícia } \\
\text { Universidade Católica do Rio de Janeiro }\end{array}$ & 9 & 9 \\
\hline $\begin{array}{c}\text { Universidade São Francisco (USF) - Universidade } \\
\text { Federal do Rio Grande do Sul (UFRGS) }\end{array}$ & 16,6 & 0 \\
\hline $\begin{array}{l}\text { Pontifícia Universidade Católica do Rio de Janeiro - } \\
\text { Universidade Federal do Rio Grande do Sul (UFRGS) }\end{array}$ & 7,6 & 0 \\
\hline MÉDIA & 13,7 & 9,6 \\
\hline
\end{tabular}


Busca: Título 'biologia'; ano de publicação '1999'

Livro encontrado na busca: JUNQUEIRA, L. C. U.; CARNEIRO, J. Histologia basica. 9.ed. Rio de Janeiro: Guanabara Koogan, 1999.

Quadro 3. Ensaio 3 - "Biologia".

\begin{tabular}{|c|c|c|}
\hline BIBLIOTECAS & ASSUNTO & FERRAMENTAS UTILIZADAS \\
\hline $\begin{array}{l}\text { Universidade da Região do Vale do } \\
\text { Rio dos Sinos (UNISINOS) }\end{array}$ & $\begin{array}{c}\text { Histologia } \\
\text { Histologia-anatomia }\end{array}$ & $\begin{array}{c}\text { Tesauro da Biblioteca Nacional e Library } \\
\text { of Congress Subject Headings }\end{array}$ \\
\hline $\begin{array}{l}\text { Universidade Federal de Pelotas } \\
\text { (UFPel) }\end{array}$ & $\begin{array}{c}\text { BiologiaHistologiaTecidos } \\
\text { (Biologia) Tecido nervoso Tecido } \\
\text { conjuntivo Citoplasmas Núcleo } \\
\text { celular Células (Biologia) Sistema } \\
\text { osteomuscular Cartilagem } \\
\text { Músculos Sangue Sistema } \\
\text { hematopoético Sistema tegumentar } \\
\text { Pele Aparelho respiratório } \\
\text { Aparelho urinário Sistema } \\
\text { endócrino Aparelho reprodutor } \\
\text { Órgãos dos sentidos }\end{array}$ & NÃO INFORMADO \\
\hline $\begin{array}{l}\text { Centro Universitário } \\
\text { Franciscano (UNIFRA) }\end{array}$ & $\begin{array}{l}\text { Célula } \\
\text { Histologia } \\
\text { Tecidos } \\
\text { Biologia tecidual }\end{array}$ & NÃO INFORMADO \\
\hline $\begin{array}{l}\text { Universidade Estadual de } \\
\text { Londrina (UEL) }\end{array}$ & Histologia & BIBLIODATA \\
\hline $\begin{array}{l}\text { Universidade Regional do Noroeste } \\
\text { do Estado do Rio Grande do Sul - } \\
\text { (UNIJUÍ) }\end{array}$ & $\begin{array}{c}\text { Medicina } \\
\text { Histologia } \\
\text { Tecidos } \\
\text { Sistema circulatório } \\
\text { Hemocitopoese } \\
\text { Aparelho respiratório } \\
\text { Aparelho urinário } \\
\text { (todos esses termos são não } \\
\text { controlados) }\end{array}$ & NÃO INFORMADO \\
\hline
\end{tabular}


Tabela 3. Índice de consistência entre pares de bibliotecas do Ensaio 3.

\begin{tabular}{|c|c|c|}
\hline PARES DE BIBLIOTECAS & $\begin{array}{c}\text { ÍNDICE DE } \\
\text { CONSISTÊNCIA } \\
\text { (RELAXADO) }\end{array}$ & $\begin{array}{l}\text { ÍNDICE DE } \\
\text { CONSISTÊNCIA } \\
\text { (RÍGIDO) }\end{array}$ \\
\hline $\begin{array}{c}\text { Universidade da Região do Vale do Rio dos Sinos (UNISINOS) - } \\
\text { Universidade Federal de Pelotas (UFPel) }\end{array}$ & 4,7 & 4,7 \\
\hline $\begin{array}{c}\text { Universidade da Região do Vale do Rio dos Sinos (UNISINOS) - Centro } \\
\text { Universitário Franciscano (UNIFRA) }\end{array}$ & 20 & 20 \\
\hline $\begin{array}{c}\text { Universidade da Região do Vale do Rio dos Sinos (UNISINOS) - } \\
\text { Universidade Estadual de Londrina (UEL) }\end{array}$ & 50 & 50 \\
\hline $\begin{array}{l}\text { Universidade da Região do Vale do Rio dos Sinos (UNISINOS) - } \\
\text { Universidade Regional do Noroeste do Estado do Rio Grande do SUI (UNIJUI) }\end{array}$ & 12,5 & 12,5 \\
\hline $\begin{array}{c}\text { Universidade Federal de Pelotas (UFPel) - Centro Universitário } \\
\text { Franciscano (UNIFRA) }\end{array}$ & 14,2 & 9 \\
\hline $\begin{array}{c}\text { Universidade Federal de Pelotas (UFPel) - Universidade Estadual de } \\
\text { Londrina (UEL) }\end{array}$ & 5 & 5 \\
\hline $\begin{array}{c}\text { Universidade Federal de Pelotas (UFPel) - Universidade Regional do } \\
\text { Noroeste do Estado do Rio Grande do Sul (UNIJUÍ) }\end{array}$ & 17,3 & 17,3 \\
\hline $\begin{array}{c}\text { Centro Universitário Franciscano (UNIFRA) - Universidade Estadual de } \\
\text { Londrina (UEL) }\end{array}$ & 25 & 25 \\
\hline $\begin{array}{c}\text { Centro Universitário Franciscano (UNIFRA) - Universidade Regional do } \\
\text { Noroeste do Estado do Rio Grande do Sul (UNIJUÍ) }\end{array}$ & 22,2 & 22,2 \\
\hline $\begin{array}{c}\text { Universidade Estadual de Londrina (UEL) - Universidade Regional do } \\
\text { Noroeste do Estado do Rio Grande do Sul (UNIJUI) }\end{array}$ & 14,2 & 14,2 \\
\hline MÉDIA & 18,5 & 17,9 \\
\hline
\end{tabular}


Busca: Título 'anatomia; ano de publicação '2000'.

Livro encontrado na busca: SOBOTTA, J. Atlas de anatomia humana. 21. ed. Rio de Janeiro: Guanabara Koogan. 2000. 2 v.

Quadro 4. Ensaio 4 - "Anatomia".

\begin{tabular}{|c|c|c|}
\hline BIBLIOTECAS & ASSUNTO & FERRAMENTAS UTILIZADAS \\
\hline $\begin{array}{c}\text { Universidade Federal do Paraná } \\
\text { (UFPR) }\end{array}$ & Anatomia humana - Atlas & BIBLIODATA \\
\hline $\begin{array}{c}\text { Universidade Estadual do } \\
\text { Centro-Oeste (UNICENTRO) }\end{array}$ & $\begin{array}{c}\text { Anatomia humana } \\
\text { Medicina }\end{array}$ & Library of Congress Subject Headings \\
\hline $\begin{array}{c}\text { Pontifícia Universidade Católica } \\
\text { (PUC - PR) }\end{array}$ & Anatomia humana - Atlas & NÃO INFORMADO \\
\hline $\begin{array}{c}\text { Universidade Estadual de } \\
\text { Maringá (UEM) }\end{array}$ & Anatomia humana - Atlas & NÃO INFORMADO \\
\hline $\begin{array}{c}\text { Universidade Regional de } \\
\text { Blumenau (FURB/SC) }\end{array}$ & Anatomia humana - Atlas & \\
\hline
\end{tabular}

Tabela 4. Índice de consistência entre pares de bibliotecas do Ensaio 4.

\begin{tabular}{|c|c|c|}
\hline PARES DE BIBLIOTECAS & $\begin{array}{c}\text { ÍNDICE DE } \\
\text { CONSISTÊNCIA } \\
\text { (RELAXADO) }\end{array}$ & $\begin{array}{c}\text { ÍNDICE DE } \\
\text { CONSISTÊNCIA } \\
\text { (RÍGIDO) }\end{array}$ \\
\hline $\begin{array}{c}\text { Universidade Federal do Paraná (UFPR) - } \\
\text { Universidade Estadual do Centro-Oeste (UNICENTRO) }\end{array}$ & 33,3 & 0 \\
\hline $\begin{array}{l}\text { Universidade Federal do Paraná (UFPR) - } \\
\text { Pontifícia Universidade Católica (PUC - PR) }\end{array}$ & 100 & 100 \\
\hline $\begin{array}{l}\text { Universidade Federal do Paraná (UFPR) - } \\
\text { Universidade Estadual de Maringá (UEM) }\end{array}$ & 100 & 100 \\
\hline $\begin{array}{c}\text { Universidade Federal do Paraná (UFPR) - } \\
\text { Universidade Regional de Blumenau (FURB/SC) }\end{array}$ & 100 & 100 \\
\hline $\begin{array}{l}\text { Universidade Estadual do Centro-Oeste (UNICENTRO) - } \\
\text { Pontifícia Universidade Católica (PUC - PR) }\end{array}$ & 33,3 & 0 \\
\hline $\begin{array}{c}\text { Universidade Estadual do Centro-Oeste (UNICENTRO) - } \\
\text { Universidade Estadual de Maringá (UEM) }\end{array}$ & 33,3 & 0 \\
\hline $\begin{array}{c}\text { Universidade Estadual do Centro-Oeste (UNICENTRO) - } \\
\text { Universidade Regional de Blumenau (FURB/SC) }\end{array}$ & 33,3 & 0 \\
\hline $\begin{array}{c}\text { Pontifícia Universidade Católica (PUC - PR) - } \\
\text { Universidade Estadual de Maringá (UEM) }\end{array}$ & 100 & 100 \\
\hline $\begin{array}{l}\text { Pontifícia Universidade Católica (PUC - PR) - } \\
\text { Universidade Regional de Blumenau (FURB/SC) }\end{array}$ & 100 & 100 \\
\hline $\begin{array}{l}\text { Universidade Estadual de Maringá (UEM) - } \\
\text { Universidade Regional de Blumenau (FURB/SC) }\end{array}$ & 100 & 100 \\
\hline MÉDIA & 73,3 & 60 \\
\hline
\end{tabular}




\section{ENSAIO 5}

Busca: Título 'química; ano de publicação '2001'.

Livro encontrado na busca: ATKINS, P.W.; JONES, L. Princípios de química: questionando a vida moderna e o meio ambiente. Porto Alegre: Bookman, 2001.

Quadro 5. Ensaio 5 - "Química".

\begin{tabular}{|c|c|c|}
\hline BIBLIOTECAS & ASSUNTO & FERRAMENTAS UTILIZADAS \\
\hline $\begin{array}{c}\text { Universidade Federal de Santa } \\
\text { Catarina (UFSC) }\end{array}$ & $\begin{array}{c}\text { Química } \\
\text { Química inorgânica }\end{array}$ & BIBLIODATA \\
\hline $\begin{array}{c}\text { Universidade do Estado de Santa } \\
\text { Catarina - (UDESC) }\end{array}$ & Química & NÃO INFORMADO \\
\hline $\begin{array}{c}\text { Fundação das Escolas Unidas do } \\
\text { Planalto Catarinense (UNIPLAC) }\end{array}$ & Química - estudo e ensino & Não utiliza nenhuma linguagem \\
\hline $\begin{array}{c}\text { Universidade Federal do Rio } \\
\text { Grande do Sul (UFRGS) }\end{array}$ & $\begin{array}{c}\text { Química } \\
\text { Enímica: problemas }\end{array}$ & NÃo INFOrior \\
\hline $\begin{array}{c}\text { Universidade de Caxias do Sul } \\
\text { (UCS) }\end{array}$ & Química & \\
\hline
\end{tabular}

Tabela 5. Índice de consistência entre pares de bibliotecas do Ensaio 5.

\section{PARES DE BIBLIOTECAS}

\begin{tabular}{|c|c|c|}
\hline $\begin{array}{l}\text { Universidade Federal de Santa Catarina (UFSC) - } \\
\text { Universidade do Estado de Santa Catarina (UDESC) }\end{array}$ & 50 & 50 \\
\hline $\begin{array}{c}\text { Universidade Federal de Santa Catarina (UFSC) - } \\
\text { Fundação das Escolas Unidas do Planalto Catarinense (UNIPLAC) }\end{array}$ & 20 & 0 \\
\hline $\begin{array}{l}\text { Universidade Federal de Santa Catarina (UFSC) - } \\
\text { Universidade Federal do Rio Grande do Sul (UFRGS) }\end{array}$ & 25 & 25 \\
\hline $\begin{array}{c}\text { Universidade Federal de Santa Catarina (UFSC) - } \\
\text { Universidade de Caxias do Sul (UCS) }\end{array}$ & 50 & 50 \\
\hline $\begin{array}{l}\text { Universidade do Estado de Santa Catarina (UDESC) - } \\
\text { Fundação das Escolas Unidas do Planalto Catarinense (UNIPLAC) }\end{array}$ & 33,3 & 0 \\
\hline $\begin{array}{l}\text { Universidade do Estado de Santa Catarina (UDESC) - } \\
\text { Universidade Federal do Rio Grande do Sul (UFRGS) }\end{array}$ & 33,3 & 33,3 \\
\hline $\begin{array}{c}\text { Universidade do Estado de Santa Catarina (UDESC) - } \\
\text { Universidade de Caxias do Sul (UCS) }\end{array}$ & 100 & 100 \\
\hline $\begin{array}{c}\text { Fundação das Escolas Unidas do Planalto Catarinense (UNIPLAC) - } \\
\text { Universidade Federal do Rio Grande do Sul (UFRGS) }\end{array}$ & 14,2 & 0 \\
\hline $\begin{array}{c}\text { Fundação das Escolas Unidas do Planalto Catarinense (UNIPLAC) - } \\
\text { Universidade de Caxias do Sul (UCS) }\end{array}$ & 33,3 & 0 \\
\hline $\begin{array}{c}\text { Universidade Federal do Rio Grande do Sul (UFRGS) - } \\
\text { Universidade de Caxias do Sul (UCS) }\end{array}$ & 33,3 & 33,3 \\
\hline MÉDIA & 39,2 & 29,1 \\
\hline
\end{tabular}


Busca: Título 'educar; ano de publicação '2002'.

Livro encontrado na busca: GIKOVATE, F. A arte de educar. Curitiba: Nova Didática, 2002.

Quadro 6. Ensaio 6 - "Educar".

\begin{tabular}{|c|c|c|}
\hline BIBLIOTECAS & ASSUNTO & FERRAMENTAS UTILIZADAS \\
\hline $\begin{array}{c}\text { Universidade Regional de } \\
\text { Blumenau (FURB/SC) }\end{array}$ & Educação - Filosofia & NÃO INFORMADO \\
\hline $\begin{array}{c}\text { Universidade de Caxias do Sul } \\
\text { (UCS) }\end{array}$ & $\begin{array}{c}\text { Educação - Aspectos psicológicos } \\
\text { Psicologia educacional - Educadores } \\
\text { Formação profissional - Psicologia }\end{array}$ & NÃO INFORMADO \\
\hline $\begin{array}{c}\text { Universidade Federal de } \\
\text { Uberlândia (UFU) }\end{array}$ & $\begin{array}{c}\text { Educação - Filosofia } \\
\text { Universidade de São Paulo (USP) }\end{array}$ & $\begin{array}{c}\text { Ensino e aprendizagem } \\
\text { Psicopedagogia } \\
\text { Professores }\end{array}$ \\
\hline $\begin{array}{c}\text { Universidade Federal do Paraná } \\
\text { (UFPR) }\end{array}$ & Educação - Brasil & Vocabulário Controlado \\
\hline
\end{tabular}

Tabela 6. Índice de consistência entre pares de bibliotecas do Ensaio 6.

\begin{tabular}{|c|c|c|}
\hline PARES DE BIBLIOTECAS & $\begin{array}{c}\text { ÍNDICE DE } \\
\text { CONSISTÊNCIA } \\
\text { (RELAXADO) }\end{array}$ & $\begin{array}{c}\text { ÍNDICE DE } \\
\text { CONSISTÊNCIA } \\
\text { (RÍGIDO) }\end{array}$ \\
\hline $\begin{array}{c}\text { Universidade Regional de Blumenau (FURB/SC) - } \\
\text { Universidade de Caxias do Sul (UCS) }\end{array}$ & 14,2 & 0 \\
\hline $\begin{array}{c}\text { Universidade Regional de Blumenau (FURB/SC) - } \\
\text { Universidade Federal de Uberlândia }\end{array}$ & 100 & 100 \\
\hline $\begin{array}{c}\text { Universidade Regional de Blumenau (FURB/SC) - } \\
\text { Universidade de São Paulo (USP) }\end{array}$ & 0 & 0 \\
\hline $\begin{array}{c}\text { Universidade Regional de Blumenau (FURB/SC) - } \\
\text { Universidade Federal do Paraná (UFPR) }\end{array}$ & 33,3 & 0 \\
\hline $\begin{array}{l}\text { Universidade de Caxias do Sul (UCS) - } \\
\text { Universidade Federal de Uberlândia }\end{array}$ & 14,2 & 0 \\
\hline $\begin{array}{l}\text { Universidade de Caxias do Sul (UCS) - } \\
\text { Universidade de São Paulo (USP) }\end{array}$ & 9 & 0 \\
\hline $\begin{array}{l}\text { Universidade de Caxias do Sul (UCS) - } \\
\text { Universidade Federal do Paraná (UFPR) }\end{array}$ & 14,2 & 0 \\
\hline $\begin{array}{l}\text { Universidade Federal de Uberlândia - } \\
\text { Universidade de São Paulo (USP) }\end{array}$ & 0 & 0 \\
\hline $\begin{array}{l}\text { Universidade Federal de Uberlândia - } \\
\text { Universidade Federal do Paraná (UFPR) }\end{array}$ & 33,3 & 0 \\
\hline $\begin{array}{l}\text { Universidade de São Paulo (USP) - } \\
\text { Universidade Federal do Paraná (UFPR) }\end{array}$ & 0 & 0 \\
\hline MÉDIA & 21,8 & 10 \\
\hline
\end{tabular}




\section{ENSAIO 7}

Busca: Título 'engenharia; ano de publicação '2003'.

Livro encontrado na busca: OGATA, K. Engenharia de controle moderno. 4. ed. São Paulo: Prentice-Hall, 2003.

Quadro 7. Ensaio 7 - "Engenharia".

\begin{tabular}{|c|c|c|}
\hline BIBLIOTECAS & ASSUNTO & FERRAMENTAS UTILIZADAS \\
\hline Universidade São Francisco (USF) & $\begin{array}{c}\text { MATLAB (Programa de computador) } \\
\text { Controle automático } \\
\text { Engenharia } \\
\text { Engenharia elétrica }\end{array}$ & NÃO INFORMADO \\
\hline $\begin{array}{c}\text { Universidade Estadual de } \\
\text { Campinas (UNICAMP) }\end{array}$ & $\begin{array}{c}\text { Controle automático } \\
\text { Teoria de controle }\end{array}$ & BIBLIODATA \\
\hline $\begin{array}{c}\text { Pontifícia Universidade Católica } \\
\text { de Campinas - (PUC-Campinas) }\end{array}$ & $\begin{array}{c}\text { Controle automático } \\
\text { Universidade Estadual Paulista } \\
\text { Júlio de Mesquita Filho (UNESP) }\end{array}$ & $\begin{array}{c}\text { Controle automáticoTeoria do } \\
\text { controleSistemas de controle por } \\
\text { realimentação }\end{array}$ \\
\hline Universidade de São Paulo (USP) & $\begin{array}{c}\text { Sistemas de controle } \\
\text { Controle automático }\end{array}$ & Subject Headings e APA \\
\hline
\end{tabular}

Tabela 7. Índice de consistência entre pares de bibliotecas do Ensaio 7.

\begin{tabular}{|c|c|c|}
\hline PARES DE BIBLIOTECAS & $\begin{array}{c}\text { ÍNDICE DE } \\
\text { CONSISTÊNCIA } \\
\text { (RELAXADO) }\end{array}$ & $\begin{array}{c}\text { ÍNDICE DE } \\
\text { CONSISTÊNCIA } \\
\text { (RÍGIDO) }\end{array}$ \\
\hline $\begin{array}{c}\text { Universidade São Francisco (USF) - } \\
\text { Universidade Estadual de Campinas (UNICAMP) }\end{array}$ & 20 & 20 \\
\hline $\begin{array}{l}\text { Universidade São Francisco (USF) Universidade Estadual } \\
\text { de Campinas (UNICAMP) - Pontifícia Universidade }\end{array}$ & 25 & 25 \\
\hline $\begin{array}{c}\text { Católica de Campinas - (PUC-Campinas) } \\
\text { Universidade São Francisco (USF) - }\end{array}$ & 16,6 & 16,6 \\
\hline $\begin{array}{l}\text { Universidade Estadual Paulista Júlio de Mesquita Filho (UNESP) } \\
\text { Universidade São Francisco (USF) - Universidade de São Paulo (USP) }\end{array}$ & 20 & 20 \\
\hline $\begin{array}{l}\text { Universidade Estadual de Campinas (UNICAMP) - } \\
\text { Pontifícia Universidade Católica de Campinas - (PUC-Campinas) }\end{array}$ & 50 & 50 \\
\hline $\begin{array}{l}\text { Universidade Estadual de Campinas (UNICAMP) - } \\
\text { Universidade Estadual Paulista Júlio de Mesquita Filho (UNESP) }\end{array}$ & 42,8 & 25 \\
\hline $\begin{array}{c}\text { Universidade Estadual de Campinas (UNICAMP) - } \\
\text { Universidade de São Paulo (USP) }\end{array}$ & 60 & 60 \\
\hline $\begin{array}{l}\text { Pontifícia Universidade Católica de Campinas - (PUC-Campinas) - } \\
\text { Universidade Estadual Paulista Júlio de Mesquita Filho (UNESP) }\end{array}$ & 33,3 & 33,3 \\
\hline $\begin{array}{c}\text { Pontifícia Universidade Católica de Campinas - (PUC-Campinas) - } \\
\text { Universidade de São Paulo (USP) }\end{array}$ & 50 & 50 \\
\hline $\begin{array}{c}\text { Universidade Estadual Paulista Júlio de Mesquita Filho (UNESP) - } \\
\text { Universidade de São Paulo (USP) }\end{array}$ & 42,8 & 25 \\
\hline MÉDIA & 36 & 32,4 \\
\hline
\end{tabular}


Busca: Título 'estatística; ano de publicação '2004'.

Livro encontrado na busca: BARBETTA, Pedro Alberto. Estatística para cursos de engenharia e informática. São Paulo: Atlas, 2004.

Quadro 8. Ensaio 8 - "Estatística".

\begin{tabular}{|c|c|c|}
\hline BIBLIOTECAS & ASSUNTO & FERRAMENTAS UTILIZADAS \\
\hline $\begin{array}{c}\text { Universidade Estadual do Centro- } \\
\text { Oeste (UNICENTRO) }\end{array}$ & $\begin{array}{l}\text { Estatística - engenharia } \\
\text { Estatistíca - informatica }\end{array}$ & NÃO INFORMADO \\
\hline $\begin{array}{l}\text { Universidade do Estado de Santa } \\
\text { Catarina (UDESC) }\end{array}$ & $\begin{array}{c}\text { Engenharia - Métodos estatísticos } \\
\text { Informática - Métodos estatísticos } \\
\text { Estatística matemática } \\
\text { Pesquisa científica }\end{array}$ & BIBLIODATA \\
\hline $\begin{array}{l}\text { Universidade Federal de Santa } \\
\text { Maria }\end{array}$ & $\begin{array}{c}\text { Matemática } \\
\text { Estatística } \\
\text { Estatística aplicada } \\
\text { Estatística matemática } \\
\text { Engenharia } \\
\text { Informática } \\
\text { Computação }\end{array}$ & NÃO INFORMADO \\
\hline $\begin{array}{l}\text { Universidade Federal de Viçosa } \\
\text { (UFV) }\end{array}$ & Estatística & NÃO INFORMADO \\
\hline $\begin{array}{l}\text { Universidade Estadual Paulista } \\
\text { Júlio de Mesquita Filho (UNESP) }\end{array}$ & $\begin{array}{c}\text { Engenharia - métodos estatísticos } \\
\text { Informática - métodos estatísticos } \\
\text { Estatística matemática }\end{array}$ & BIBLIODATA \\
\hline
\end{tabular}

Quadro 8. Índice de consistência entre pares de bibliotecas do Ensaio 8.

\section{PARES DE BIBLIOTECAS}

\begin{tabular}{|c|c|c|}
\hline & 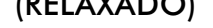 & (RIGIDU) \\
\hline $\begin{array}{l}\text { Universidade Estadual do Centro-Oeste (UNICENTRO) - } \\
\text { Universidade do Estado de Santa Catarina (UDESC) }\end{array}$ & 20 & 0 \\
\hline $\begin{array}{l}\text { Universidade Estadual do Centro-Oeste (UNICENTRO) - } \\
\text { Universidade Federal de Santa Maria }\end{array}$ & 20 & 0 \\
\hline $\begin{array}{c}\text { Universidade Estadual do Centro-Oeste (UNICENTRO) - } \\
\text { Universidade Federal de Viçosa (UFV) }\end{array}$ & 20 & 0 \\
\hline $\begin{array}{l}\text { Universidade Estadual do Centro-Oeste (UNICENTRO) - } \\
\text { Universidade Estadual Paulista Júlio de Mesquita Filho (UNESP) }\end{array}$ & 20 & 0 \\
\hline $\begin{array}{c}\text { Universidade do Estado de Santa Catarina (UDESC) - } \\
\text { Universidade Federal de Santa Maria }\end{array}$ & 18,1 & 0 \\
\hline $\begin{array}{c}\text { Universidade do Estado de Santa Catarina (UDESC) - } \\
\text { Universidade Federal de Viçosa (UFV) }\end{array}$ & 11,1 & 0 \\
\hline $\begin{array}{l}\text { Universidade do Estado de Santa Catarina (UDESC) - } \\
\text { Universidade Estadual Paulista Júlio de Mesquita Filho (UNESP) }\end{array}$ & 75 & 75 \\
\hline $\begin{array}{l}\text { Universidade Federal de Santa Maria - } \\
\text { Universidade Federal de Viçosa (UFV) }\end{array}$ & 14,2 & 14,2 \\
\hline $\begin{array}{c}\text { Universidade Federal de Santa Maria - } \\
\text { Universidade Estadual Paulista Júlio de Mesquita Filho (UNESP) }\end{array}$ & 25 & 11,1 \\
\hline $\begin{array}{c}\text { Universidade Federal de Viçosa (UFV) - } \\
\text { Universidade Estadual Paulista Júlio de Mesquita Filho (UNESP) }\end{array}$ & 14,2 & 0 \\
\hline MÉDIA & 23,7 & 10 \\
\hline
\end{tabular}




\section{ENSAIO 9}

Busca: Título 'zahir'; ano de publicação '2005'.

Livro encontrado na busca: COELHO, P. O Zahir. Rio de Janeiro: Rocco, 2005.

Quadro 9. Ensaio 9 - "Zahir".

\begin{tabular}{|c|c|c|}
\hline BIBLIOTECAS & ASSUNTO & FERRAMENTAS UTILIZADAS \\
\hline $\begin{array}{c}\text { Universidade Federal de Santa } \\
\text { Maria }\end{array}$ & $\begin{array}{c}\text { Literatura } \\
\text { Literatura brasileira } \\
\text { Romance }\end{array}$ & NÃO INFORMADO \\
\hline $\begin{array}{c}\text { Universidade Federal de Viçosa } \\
\text { (UFV) }\end{array}$ & $\begin{array}{c}\text { Ficção brasileira } \\
\text { Literatura brasileira }\end{array}$ & Tesauro da Biblioteca Nacional e DeCS \\
\hline $\begin{array}{c}\text { Pontifícia Universidade Católica } \\
\text { de Minas Gerais (PUC-MG) }\end{array}$ & Ficção brasileira & NÃO INFORMADO \\
\hline $\begin{array}{c}\text { Universidade Federal de Minas } \\
\text { Gerais (UFMG) }\end{array}$ & $\begin{array}{c}\text { Literatura brasileira } \\
\text { Romance brasileiro }\end{array}$ & $\begin{array}{c}\text { BIBLIODATA, DeCS, Tesauro da } \\
\text { Biblioteca Nacional e Library of } \\
\text { Congress Subject Headings }\end{array}$ \\
\hline $\begin{array}{c}\text { Universidade Federal de } \\
\text { Uberlândia (UFU) }\end{array}$ & Ficção brasileira & \\
\hline
\end{tabular}

Tabela 9. Índice de consistência entre pares de bibliotecas do ensaio 9.

PARES DE BIBLIOTECAS

\begin{tabular}{|c|c|}
\hline $\begin{array}{c}\text { ÍNDICE DE } \\
\text { CONSISTÊNCIA } \\
\text { (RELAXADO) }\end{array}$ & $\begin{array}{c}\text { ÍNDICE DE } \\
\text { CONSISTÊNCIA } \\
\text { (RÍGIDO) }\end{array}$ \\
\hline 25 & 25 \\
\hline 14,2 & 0 \\
\hline 42,8 & 25 \\
\hline 14,2 & 0 \\
\hline 50 & 50 \\
\hline 33,3 & 33,3 \\
\hline 50 & 50 \\
\hline 20 & 0 \\
\hline 100 & 100 \\
\hline 20 & 0 \\
\hline 36,9 & 28,3 \\
\hline
\end{tabular}


Busca: Título 'educação física'; ano de publicação '2006'.

Livro encontrado na busca: NEIRA, M. G. Educação física: desenvolvendo competências. 2. ed. São Paulo: Phorte, 2006.

Quadro 10. Ensaio 10 - "Educação Física".

\begin{tabular}{|c|c|c|}
\hline BIBLIOTECAS & ASSUNTO & FERRAMENTAS UTILIZADAS \\
\hline $\begin{array}{c}\text { Universidade Federal do Espírito } \\
\text { Santo }\end{array}$ & $\begin{array}{c}\text { Educação física - Estudo e ensino } \\
\text { Professores - Educação física }\end{array}$ & BIBLIODATA \\
\hline $\begin{array}{c}\text { Centro Universitário da Cidade } \\
\text { Professores de educação física } \\
\text { Campiversidade Estadual de }\end{array}$ & Educação física - Estudo e ensino & BIBLIODATA \\
\hline $\begin{array}{c}\text { Universidade Federal de Minas } \\
\text { Gerais (UFMG) }\end{array}$ & Educação física - Estudo e ensino & NÃO INFORMADO \\
\hline $\begin{array}{c}\text { Universidade Regional do } \\
\text { Noroeste do Estado do Rio } \\
\text { Grande do Sul (UNIJUÍ) }\end{array}$ & $\begin{array}{c}\text { Educação física } \\
\text { Ensino fundamental } \\
\text { Proposta pedagógica } \\
\text { Ensino }\end{array}$ & NÃO INFORMADO \\
\hline
\end{tabular}

Tabela 10. Índice de consistência entre pares de bibliotecas do ensaio 10.

\begin{tabular}{|c|c|c|}
\hline PARES DE BIBLIOTECAS & $\begin{array}{c}\text { ÍNDICE DE } \\
\text { CONSISTÊNCIA } \\
\text { (RELAXADO) }\end{array}$ & $\begin{array}{c}\text { ÍNDICE DE } \\
\text { CONSISTÊNCIA } \\
\text { (RÍGIDO) }\end{array}$ \\
\hline $\begin{array}{l}\text { Universidade Federal do Espírito Santo - } \\
\text { Centro Universitário da Cidade }\end{array}$ & 20 & 0 \\
\hline $\begin{array}{l}\text { Universidade Federal do Espírito Santo - } \\
\text { Universidade Estadual de Campinas (UNICAMP) }\end{array}$ & 50 & 50 \\
\hline $\begin{array}{c}\text { Universidade Federal do Espírito Santo - } \\
\text { Universidade Federal de Minas Gerais (UFMG) }\end{array}$ & 50 & 50 \\
\hline $\begin{array}{c}\text { Universidade Federal do Espírito Santo - Universidade Regional do } \\
\text { Noroeste do Estado do Rio Grande do Sul (UNIJUII) }\end{array}$ & 9 & 0 \\
\hline $\begin{array}{l}\text { Centro Universitário da Cidade - } \\
\text { Universidade Estadual de Campinas (UNICAMP) }\end{array}$ & 0 & 0 \\
\hline $\begin{array}{c}\text { Centro Universitário da Cidade - } \\
\text { Universidade Federal de Minas Gerais (UFMG) }\end{array}$ & 0 & 0 \\
\hline $\begin{array}{c}\text { Centro Universitário da Cidade - Universidade Regional do Noroeste do } \\
\text { Estado do Rio Grande do Sul (UNIJUÍ) }\end{array}$ & 0 & 0 \\
\hline $\begin{array}{l}\text { Universidade Estadual de Campinas (UNICAMP) - } \\
\text { Universidade Federal de Minas Gerais (UFMG) }\end{array}$ & 100 & 100 \\
\hline $\begin{array}{c}\text { Universidade Estadual de Campinas (UNICAMP) - Universidade Regional } \\
\text { do Noroeste do Estado do Rio Grande do Sul (UNIJUÍ) }\end{array}$ & 11,1 & 0 \\
\hline $\begin{array}{l}\text { Universidade Federal de Minas Gerais (UFMG) - Universidade Regional } \\
\text { do Noroeste do Estado do Rio Grande do Sul (UNIJUÍ) }\end{array}$ & 11,1 & 0 \\
\hline MÉDIA & 25,1 & 20 \\
\hline
\end{tabular}


